\title{
Techniques for Expanding the Card Catalog of a Large Library
}

Miss Terry is catalog librarian, Columbia University Libraries.

$\mathrm{T}$ HE PRESENT account is concerned with methods of redistributing a large university library card catalog, and centers around a specific situation. It is hoped, however, that it will be suggestive in other cases where such large-scale operations must be performed.

The problem was this: the union catalog (known as the general catalog) of the Columbia University Libraries-some $3,300,000$ cards housed in 2660 trays-was to be expanded by redistribution into a total of 4I3O trays by the addition of 1470 trays originally sheltering part of the depository catalog. Before the move was effected, the decision to set up a public serials catalog for main entry serials cards reduced the available space to 3864 trays. This new space was at the beginning of the original catalog, requiring that it be backed up into its new quarters.

Much of the technique followed dates back to earlier moves and thus was known to be effective; at the same time, much of the success of the I950 move was a direct result of the location of present quarters. In minor catalog shifting that goes on spasmodically as soon as a catalog begins to burst at the seams, the work has been done at the catalog, using the consulting tables as the field of operations. Probably the wisest decision at this time was to do none of the work in the catalog area. Two facts conspired to make this possible: the opening of the Cataloging Department directly into the general catalog, and free space, with long worktable, chairs, and floor space for accommodation of booktrucks, at the front of the Cataloging Department.

\section{Planning the New Catalog}

A preliminary step in any catalog planning or move is to measure or estimate (from sampling) the total bulk of the catalog to be accommodated. Measuring is not excessively time-consuming, and affords an accurate view of the situation. The work of measuring may be divided between two or more groups of clerical staff members, with two persons working as a team, one doing the measuring, the other recording measurements. In measuring, cards should be brought to an upright position by supporting them at front and back of the tray; they should be held in a relatively easy position, without undue crowding. In a catalog as extensive as the one under consideration it would be helpful to have a measuring scale (in inches only) stamped on the outer right side or along the top edge of that side of each tray, to save the necessity of picking up and positioning a ruler, both in the process of estimating and of apportioning tray contents later.

The number of trays available for the expanded catalog should be recorded next. There are two considerations here: first, the total trays in the expanded catalog, and second, the trays that are to be put into immediate use. It is advantageous to leave empty trays for expansion throughout the catalog rather than to use all trays, with fewer cards in each, at the beginning. To 
follow the latter plan means that added space at a given point can be gained only by a very considerable juggling of the contents of a number of trays, while totally empty trays may be introduced easily at pressure points. Top and/or bottom trays may be held in reserve, thus giving added capacity throughout the catalog for later adjustments. Certain variations in pattern may be found desirable; one was made in this case where bookshelves come close to the catalog by leaving a vertical line of trays to separate the two areas, and a second when empty trays were accumulated at the beginning of the catalog to keep catalog users at a safe and comfortable distance from a muchneeded but powerful floor fan. Another advantage of free trays is that the arbitrary inchage set up for each tray cannot be followed absolutely, making it necessary to have reserve space on which to draw even during the move. In this library, provision must be made in each catalog case for a tray to hold a supply of temporary removal cards for staff use, and a "dummy" tray with direction Apply at Reference Desk for insertion when a tray is removed to the Cataloging Department. As pressure on catalog space increases, some of these may have to be relinquished.

Once the number of trays for immediate use has been determined, the inches of cards to go into each tray can be fixed mathematically. Actually, no such arbitrary pattern can be followed in the expansion, since tray contents are dependent on beginning and ending each tray at a logical breaking point and on the probability of slow or rapid growth of a given main entry or subject group. For this reason, expanding the catalog must be regarded as a top-level rather than a clerical function.

\section{Labels for the Expanded Catalog}

(Explanatory: The label-holders in use are $5 \frac{1}{8} \times 1 \frac{3}{4}$ inches carrying two labels within one frame. The smaller label, at the left, is ${ }_{I} 11 / 16 \times I_{1}^{1}$, and shows the case number above the tray number, as $\frac{1}{110}$. The second label shows the limits, alphabetically speaking, of tray contents. This legend is apt to be rather complex; and no method of marking, save by hand printing, has been found satisfactory.)

Estimates of the number of labels required in each size for the expanded catalog should be determined early, samples of stock obtained, orders placed, and stock cut to size. Care must be taken to see that size samples are true. Number labels from the old catalog may be used, at least temporarily, as far as they will serve. For that reason, the first number labels to be made should be those with new case numbers, going back, when these are done, to replacement labels, if needed, for cases of the original catalog. Empty trays, as well as those in active use, should carry number labels.

Ordinarily we have several clerical staff members who do good lettering and numbers. They should be started on number labels well in advance of the move, giving time each day to the work. It is not necessary to limit the work to one person, although it is desirable to assign each person an area of responsibility so that lettering is uniform over a considerable space. An effort should be made to have workers adopt a straightforward style that harmonizes as far as possible. Uniform ink, preferably India, should be used.

At this time, all labels are covered with transparent Perma film (clearseal). This comes in large sheets. By laying these on a flat surface, adhesive side up, the labels may be pressed face down with just enough space between for cutting purposes. After they have adhered, the cutting process begins. Usually labels have to be trimmed somewhat in inserting into the label holders. After the 1950 move, all labels were given 
a dab of rubber cement to hold them in place, thus giving a more tidy appearance to the catalog. The method has not been entirely effective, since a considerable number of labels have already required a second treatment; this may mean only that we were too conservative in applying the cement.

Temporary labels to show tray contents were cut from a cheaper grade of stock in the 1950 move. These were in use for some weeks before permanent lettering could be done. The temporary labels were printed hastily in ink and used without protective covering.

\section{Mechanics of Expansion}

The original catalog should be moved forward or backward, depending on the location of the new trays in relation to the original catalog, so that free space will be available at the beginning of the catalog. As this repositioning is done, free trays should be arranged throughout, according to the pattern determined for the finished catalog, with pre-determined number of additional empty trays in each case to meet the expansion requirements. This should be done without disturbing tray contents, when possible. If new equipment requires leaving trays newly acquired in the cases to which they belong, contents should be carefully and rapidly transferred. Empty trays should be piled in space determined upon until such time as space is free for their insertion at the beginning of the cata$\log$, if the catalog is being moved forward.

If trays that have been in use are to be dusted and/or washed, provision should be made in ample time, since the task is a slow one. In the case of our 1950 move, this process continued for weeks. Trays were trucked into the department locker room, where running water was available. Once all empty trays are washed, those from which cards are being removed may be sub- ject to the same treatment. If so, they should be segregated. Dust cloths must be kept close at hand during the actual shifting, and a long-handled typewriter brush is effective for corners and quick dusting.

The first step in the actual moving is to fill a booktruck with trays from the catalog, beginning with the first tray of the letter A. Each truck must be checked to see that trays are in proper order and that there are no gaps due to trays not in place when the truck was filled. A record should be left at the point in the catalog from which trays are being removed: ASK AT REFERENCE DESK FOR TRAYS NOT FOUND IN THIS SECTION. Such a direction statement can be suspended by a cord or rubber band from the rod of an empty tray inserted in the vacant space.

The loaded truck was then brought to the long worktable at the front of the room where trays for expanding were assembled. A second truck was ready to receive trays on which work had been completed. Trays were returned to the catalog a truckload at a time- -15 trays to a truck -or more rapidly by hand, if there was a lag in the work.

The decision as to the amount to go into the new trays should be determined only by a qualified person, since consideration must be given to logical division and to probabilities of growth. In the 1950 move, this was carried out only by the catalog librarian and the supervisor of the Processing Unit. The procedure of the actual transfer of cards is as follows: From the back of the first tray, remove cards that are in excess of what are to be retained in the new first tray, placing these at the front of an empty tray, adding to these, from the beginning of the second tray of the old catalog, such cards as are needed to complete tray two of the new catalog. Cards then remaining in tray two of the old cata$\log$ are pushed forward and cards added from the beginning of tray three of the old 
catalog to make up tray three of the new catalog. (Complete transfer to fresh trays must be made in case used trays are to be cleaned.)

Extreme care must be taken in handling cards, since it is easy to become confused if the procedure is hurried or if the element of fatigue is involved. It has been found desirable to have the process handled by two crews-catalog librarian and clerical, and supervisor, Processing Unit, and clerical - and to limit each crew to one half day of activity each day. The clericals chosen to participate here were on the cataloging assistant level - this is the middle grade of the clerical range, and with us cataloging assistants do searching, transferring, adding of copies, changing of records, and other subprofessional duties, which make them familiar with the catalog. In the 1950 move it became evident that it was not advisable to push for a definite goal each day. When the competitive factor, either with another person or to keep to a record already established, was introduced, greater fatigue and confusion resulted. Better results came from accepting normal ups and downs.

The professional member of the crew determined the tray contents, calling out the label statement to the clerical member who recorded it on a legal size sheet in tray order. This list was used for making of permanent labels. Care must be taken to give this statement clearly and accurately. If the professional member writes the temporary label, it can be passed on as a guide for the record. In addition to making the temporary label and the sheet record, the label must be trimmed and inserted. No attempt is made here to specify who does these several tasks; it depends on the rapidity with which the process is handled and on which worker is ahead at a given moment. Some shifting about is also desirable as relief.

A protective card should be placed at front and back of each tray-this can be done by the professional member as tray contents are set up. Ordinary buff guide cards, with tab trimmed off, serve well since they are of heavier stock. Used guide cards, providing they are relatively clean, are acceptable. At a later time, the catalog should be checked to see what additional guides are required as a result of the move, particularly at the beginning of each tray of the new catalog.

Label statements should be as concise as possible and inclusive, leaving no doubt in the mind of the catalog user as to what will be found in a given tray. Each label should tell the complete story and not require looking ahead to the next tray to make sure. It may be necessary at times to adjust tray contents in order to simplify the label statement. It is easy to come up with impossible letter combinations on labels, and while it is well to avoid them, the principle of inclusiveness must be maintained, if filers are not to find themselves with a card that, as far as labels indicate, belongs nowhere. It is well to study the catalog as set up, both for good examples and for the occasional unfortunate one. The pattern to be followed should be discussed and adhered to by the two professional workers, and the sheets of label designations be edited for consistency, inclusiveness, and accuracy, preferably making the department head responsible, before permanent labels are made. Permanent labels should be inserted by the professional crew who should make a final check with temporary label and with tray contents, if necessary.

\section{Catalog Repairs}

Catalog repairs can be handled by the clerical worker as part of the procedure, although both workers should be alert in the matter. Much can be done regularly toward keeping a running check and taking

(Continued on page 252) 
will prevent any major consolidation unless a unified science library is built at some point convenient to the faculties in these subjects.

The humanities and social sciences are much in need of better library facilities and will receive first consideration in the development of building plans for the main library. These two divisions are as yet administrative conveniences rather than coordinated groups of closely related libraries. The probability that there will some day be adequate reading rooms and better coordinated acquisitional and service programs for these divisions must be an important factor in determining whether new special collections shall be permitted to grow outside the main library in subject fields which they cover.

It is too early to assert that the existing arrangement of divisions and the stipulations regarding special libraries represent a final stage in Stanford's thinking. It can only be said that they have up to this time been effective in combating some of the most serious weaknesses noted in the survey, and that within their framework there seems to be the possibility of an orderly and effective development of Stanford's collections and services.

\section{Expanding the Card Catalog}

(Continued from page 245)

prompt action. If this is done, there will be less to catch up on at the time of the move. At this time, there should be, nevertheless, an automatic inspection of trays, with elimination of any trays that are defective in body of the tray, label holder, or handle. These should be sent out regularly for repair as the work progresses.

A sufficient supply of label holder screws should be on hand, and any that turn without gripping should be replaced. In order to be effective, a replacement screw must be larger than the one replaced; old screws from the catalog should not be used under any circumstances. Our carpenter shop has been helpful in determining the size of screw needed, as well as being the source of supply.

Bent rods should be straightened; this can be done easily with a little practice. For this catalog, a supply of nuts and bolts is needed to fasten in the metal square at the back of the tray that frequently becomes detached. An extra supply of the metal part should be on hand to replace any that have been lost.

\section{Method for Complete Transfer of a Catalog}

The complete transfer of an old catalog to new equipment is in some ways less of a problem than the process of expansion. Such a procedure was experienced here when the move from Low Library to the present building took place. The planning followed the method related above, but tray contents for the new catalog were indicated by upright cards inserted at appropriate points in the original catalog. These cards carried the label statement in each instance. This preliminary work consumed a month of the time the moving of the book collections was under way. During that time, labels were made and put in place in the new catalog. When the time came for removal of the catalog to its new quarters, the process was one of simple transfer of tray contents and consumed only a few hours. It is not recalled that the catalog was used in transit, although tradition has it that encyclopedias were consulted en route. In any case, catalog trays were out of use for a brief time only. 